\title{
Genomic territories in inter-genomic hybrids: the winners and losers with hybrid fixation
}

\author{
Yerramilli Vimala ${ }^{1} \cdot$ Umesh Chandra Lavania ${ }^{2}$
}

Published online: 16 February 2021

(c) Archana Sharma Foundation of Calcutta 2021

\begin{abstract}
Genomic technologies have enabled elucidation of spatial organization of genomes and gene expression in the nucleus of the homoploid hybrids and allopolyploids. It has been shown that the participating genomes in the inter-genomic hybrid occupy definite territories in the combined nucleus forming concentric rings. The spatial position of the partner genomes is largely dependent on the relative size of their chromosomes and centromeres. In terms of genetic expression, the inter-genomic hybrids do not truly represent the combination of parental genomes, instead, there is sub-genomic dominance of one genome over the other in accordance with predisposed nucleotype. Generally, the genome with larger chromosomes surrounds the sister genome in the stable hybrid, but the genome destined to be eliminated in the unstable hybrid lie in the peripheral zone of the hybrid nucleus. The peripheral genome tends to express preferentially, but there is repression of gene expression in the surrounded genome owing to enhanced methylation / reduced transcription, making them winner or loser respectively. In a battle between the parental genomes in the unstable hybrid, the inter-genomic dosage between the participating genomes, as well as, diminution or loss of centromeric histones in the losing genome adversely affects kinetochore-microtubule assembly. The latter leads to uniparental elimination of such genome in the wide hybrids during developmental phase.
\end{abstract}

Keywords Inter-genomic hybrid · Spatial genomic organization · Genomic territories · Subgenomic dominance $\cdot$ Winners and losers in hybrid fixation

\section{Introduction}

In plants where asexual reproduction is obligatory or callus cultures where cells get open opportunity to proliferate, there are increased chances of de novo formation of heterogeneous collection of cells owing to enhanced mitotic errors. Such a behaviour is considered an important resource to realize variation even with somatic division enabling speciation in asexually propagated species [34], and evolutionary fitness of balanced genomes through passage of morphogenetic sieve [20]. In a homoploid hybrid or allopolyploid, the union of multiple genomes within one nucleus may require

Umesh Chandra Lavania

lavaniauc@yahoo.co.in

Yerramilli Vimala

yvimala17@gmail.com

1 Department of Botany, CCS University, Meerut 250004, India

2 Department of Botany, University of Lucknow, Lucknow 226 007, India new spatial arrangement of the progenitor genomes in the inter-genomic reconstitution. In the hybrid nucleotype one genome may affect the gene expression of the other [16]. Also, there may be partial or complete loss of chromosomes of one parent during developmental stabilization [12, 14, $21,29]$. Thus, the obvious question that follows is about the winners and losers in search of selection for fitness and stability in a wide hybrid. At individual cell or chromosome level it seems difficult to draw direct inferences, but could be possible if we consider whole genomes in the inter-genomic hybrids through large scale understanding of genomic territories, inter-genomic organization and character expression.

\section{Genomic territories in inter-specific hybrids}

A good number of studies have been performed on natural and synthetic inter-genomic hybrids with respect to physical positioning of the participating genomes in the hybrid nucleotype. Leitch et al. [22], based on their study undertaken on sectioned material of the manmade hybrid between Hordeum 
vulgare $\times$ Secale africanum deduced that the two genomes, contributed by different parents, tend to be spatially separated at interphase, prophase, as well as at metaphase in the wide hybrid. GISH based analysis that categorically differentiated the two genomes, further revealed that the genome originating from $H$. vulgare tended to be located more centrally than that from $S$. africanum. In another hybrid between Hordeum vulgare $\times H$. bulbosum, the H. bulbosum chromosomes were found to be located in outer periphery [33]. Similar situation about spatial disposition of ancestral genomes have been observed in the naturally occurring allopolyploid wild grass Milium montianum, where the two ancestral genomes exhibited tendency to lay apart, where M genome with small chromosomes was found to be surrounded by $\mathrm{V}$ genome with larger chromosomes [3].

An exhaustive analysis undertaken in genomic disposition on tetraploid cotton, Gossypium hirsutum, AADD (i.e. A genome of G. arboreum and D genome of G. thurberi) and synthesized tetraploid cotton, AAGG (i.e. A genome of G. arboreum and G genome of G. bickii), Han et al. [15] demonstrated that the two sub-genomes were separated in a radial pattern where small genome ( $D$ or $G$ ) tended to concentrate in center and the large genome (A) scattered along the periphery. This established that the subgenomes in the inter-specific hybrid conform to spatial genomic territory, where the genome with larger chromosome occupies peripheral territory (Fig. 1a, b). Similar pattern of spatial genomic disposition was observed in the somatic hybrids of Nicotiana $\times$ Atropa, where the genome of A. belladona with small chromosomes tended to lie at the center of the metaphase plate whereas the large chromosomes of $N$. chinensis were scattered along the periphery[13].

In a recent study on the spatial nucleus architecture in various wheat $\times$ rye and wheat $\times$ barley introgression lines it has been shown that the introgressed chromosome /chromosome arm tend to occupy discrete, separate positions in different somatic tissues during different cell cycle stages. It is further suggested that there is apparent link between the length of chromosome and spatial positioning, wherein shorter chromosomes or chromosome arms are preferentially located closer to the center of the nucleus and the longer chromosomes and chromosome arms occupy more peripheral areas of the nucleus [18].

However, in the interspecific hybrids that exhibited tendency of uniparental elimination, whether partial or complete, the genome of the parent that was destined to be
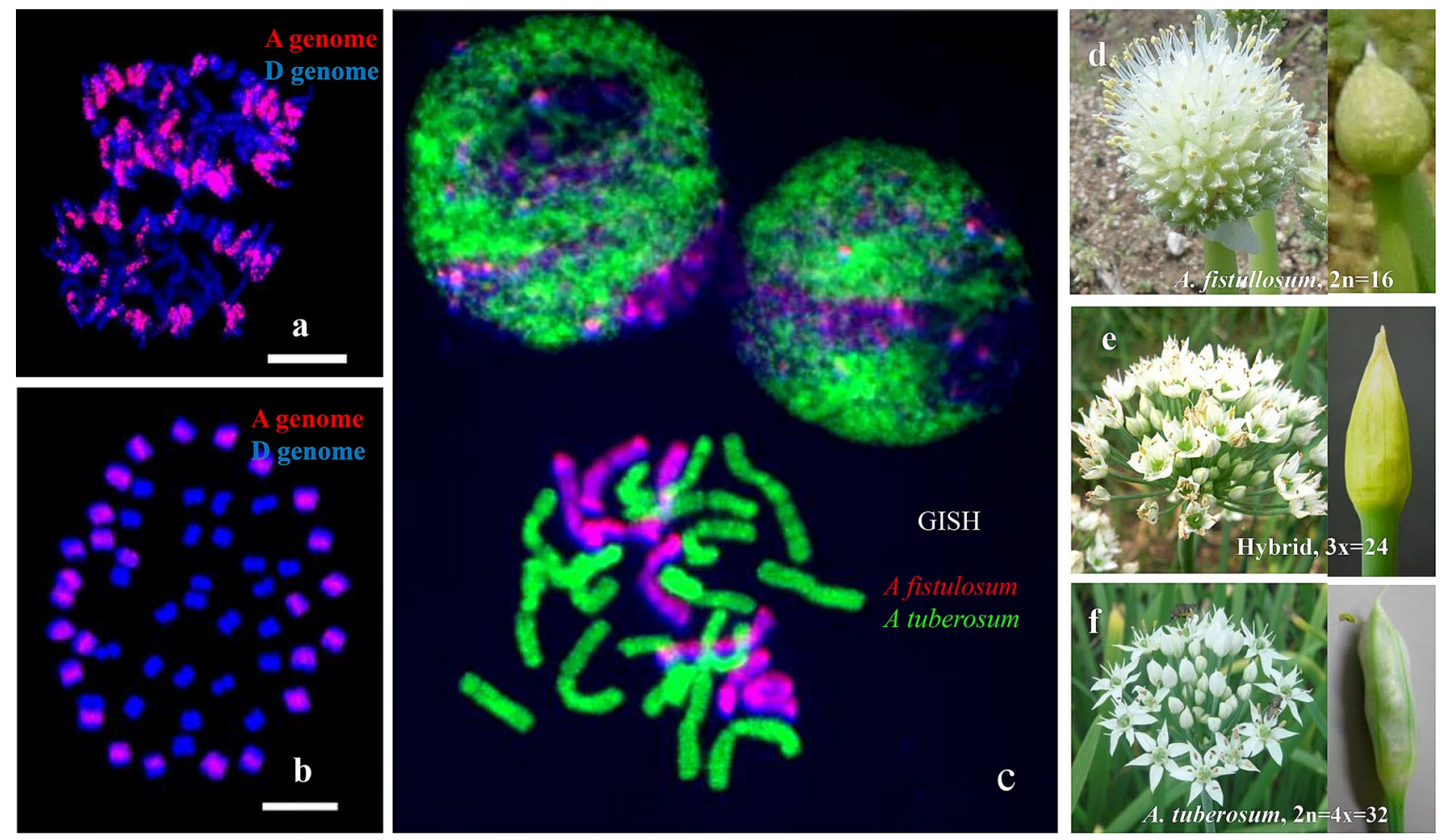

Fig. 1 GISH based genomic differentiation and their spatial orientation in the nucleus. a, b Gossypium hybrid-showing genomic territories at anaphase and metaphase of A (peripheral) and D (central) genome; c Allium hybrid-showing genomic territories at interphase and metaphase wherein A. tuberosum is peripheral and A. fistullo- sum is towards centre, $\mathbf{d}-\mathbf{f}$ Inflorescence and bud of the parent $(\mathbf{d}, \mathbf{f})$ and hybrid (e) - hybrid ' $\mathbf{e}$ ' shows more resemblance towards parent 'f' that show peripheral localization in the hybrid nucleus (c). Figure source: a, b [15], and c-f [40] 
eliminated was found to occupy peripheral position irrespective of the chromosome size. This is apparent from the observations made on the interspecific hybrids between oat $\times$ maize and wheat $\times$ pearl millet, wherein the introgressed chromatin was predominantly located at the nuclear periphery. GISH based localization of maize chromosomes in oat $\times$ maize addition lines further showed that the added maize chromosomes are preferentially positioned at the nuclear periphery [1]. The peripheral positioning of pearl millet chromatin was shown to be part of a chromosome elimination process that involved the formation of nuclear extrusions in the interphase [12]. This is in line with spatial disposition of the two genomes in the Hordeum vulgare and $H$. bulbosum hybrids, where the $H$. bulbosum chromatin destined for elimination is deposited at the nuclear edge [17].

From the above it becomes clear that the participating genomes are spatially organized in the nucleus at genome level, and there is apparent link between the chromosome size and behaviour during development that shape the nuclear architecture in the hybrid.

\section{Winners and losers in the spatial organization}

\section{Correlation of phenotype with chromosome position}

It has been observed in many interspecific hybrids that they do not reflect mid-parent appearance of the parental species, instead exhibit a form of parental dominance of one species over the other owing to different gene expression properties $[1,14]$. Finch and Bennett [10] reported that the F1 hybrid between $H$. vulgare $\times S$. africanum does not exhibit intermediate appearance of the two parents, but show resemblance with the peripherally located Secale parent for many different characters. Similarly in Triticale (a hybrid between wheat, Triticum durum $\times$ rye Secale cereale) it was observed that the hybrid does not resemble its true intermediate, but show many more features of rye, parent that has peripheral disposition. The observations suggest that in the hybrids the peripheral genome tends to be expressed preferentially [16]. Similar situation was observed in Allium hybrid (A. fistulosum, $2 n=16 \times A$. tuberosum, $2 n=32$ ), wherein hybrid exhibited more resemblance to peripherally located genome of $A$. tuberosum (Fig. 1c-f) [40]. Further, in all these instances the genome with larger chromosomes was found to be preferably positioned towards periphery in the hybrid nucleus.

\section{The subgenomic dominance}

(a) Gene Expression It is a common observation that during the course of intergenomic hybrids fixation, one of the parental genome becomes dominant over the other, a phenomenon called subgenomic dominance. At the same time, as pointed above the physical positioning of the chromosomes and genomes in the hybrid nucleotype influences genomic imprinting of one genome over the other, wherein the peripheral genome is found to exhibit subgenomic dominance in phenotypic expression. One simple explanation that could be given is that the masking effect of peripheral genome reduces the transcription factor accessibility associated with chromatin remodelling [23]. Other factor attributed to reduced gene expression in the submissive genome is methylated TEs that reduce / silence the expression in nearby gene $[1,4,14,30]$. This is consistent with an earlier observation whereby treatment with the demethylation agent azacytidine was found to release hidden variation in triticale hybrid which was lost because of genomic imprinting phenomena [16].

(b) Gene fractionation and centromere dysfunction In order to obtain a more diploid-like state, polyploid genomes will undergo gene loss (biased fractionation) [1, 8, 24, 39], DNA purging [35] and genome downsizing [14]. Furthermore, it was shown that the dominant subgenome, which retains more genes, also exhibits significantly higher overall gene expression compared to the submissive subgenome [1, 32]. As such, the less fractionated and more highly expressed subgenome is referred to as the 'dominant subgenome' and the more fractionated and lowly expressed subgenome is referred to as the 'submissive subgenome'. Collectively this phenomena is called 'subgenome dominance' [1]. In addition to just gene fractionation, in certain artificial intergenomic hybrids subgenomic dominance has been observed to the extent that the submissive subgenome is eliminated all together owing to centromere dysfunction [31].

In this respect it is worth mentioning the observations made on the structural organization of chromosomes and centromers of the participating genomes in the interspecific hybrids between Hordeum vulgare $\times H$. bulbosum, where both stable or unstable hybrids (showing gradual elimination of bulbosum chromosomes) are known to be formed depending upon genotype of the bulbosum parent and temperature of cultural environment during embryo development [2, 11]. Schwarzacher et al. [33] while reconstructing the three dimensional position of the chromosomes and centromeres in root-tip and premeiotic mitosis of the said hybrids have shown that centromeres of the parental genomes tend to lie in spatially separated domains in both tissues. Whereas, there is apparently no difference in the mean chromosome size of the two genomes, but the H. bulbosum chromosomes appear less condensed with weaker (or smaller) centromereassociated structures compared to $H$. vulgare genome with larger centromere and more condensed chromosomes. It is further observed that vulgare genome is disposed in the central zone and the bulbosum genome is more peripheral in 
the nucleotype. It was therefore suggested that the activity, rate or timing of production of the centromeric structures is likely to be under genetic control, and might be correlated with the tendency of chromosomes of particular genome to be lost in unstable hybrids [33].

(c) In the battle between parental chromosomes in the intergenomic hybrids-the centromeres hold the key: One of the important resource to understand genomic winner or loser i.e. battle between parental chromosomes in the inter-genomic hybrids is the classic example of artificial hybrids between Hordeum vulgare $\times H$. bulbosum [6]. Depending upon the genotype and gamete source of bulbosum parent, both stable and unstable hybrids could be produced. Also culture conditions with respect to temperature and nutrition during embryo rescue could help realize stability to the otherwise unstable hybrid to some extent through regulation of the cell cycle [11].

In order to elucidate the reason and mechanism behind differential behaviour of the partner genomes in the intergenomic hybrids, Sanei [31] studied the structural organization of centromeres of the stable vs unstable hybrids of $H$. vulgare $\times H$. bulbosum through immunolocalization. It has been found that in the interspecific hybrids of barley the uniparental chromosome elimination is preceded by the loss of a histone $\mathrm{H} 3$ variant known as the centromere-specific histone $\mathrm{H} 3$ variant (CENH3). The CENH3 deficiency in the $H$. bulbosum turns its centromere inactive for attachment to the spindle required for regulated chromosome segregation. This triggers uniparental elimination of bulbosum chromosomes in the unstable hybrid. Such centromere inactivity results from centromeric loss of $\mathrm{CENH} 3$ rather than uniparental silencing of CENH3 genes [31].

The elimination of the $H$. bulbosum genome in unstable hybrids is gradual, taking place over several days after pollination $[2,9]$. When chromosomes are replicated during $\mathrm{S}$ phase of the cell cycle, histones including CENH3 are distributed between the two sister chromatids [7]. These observations suggest that $H$. bulbosum chromosomes enter the zygote with a normal complement of CENH3, which is gradually depleted by several rounds of DNA replication until the kinetochore is no longer able to function. Therefore, reloading of CENH3 after DNA replication is specifically defective in $H$. bulbosum derived chromosomes, although it is normal in chromosomes from the H. vulgare parent [6].

Wang and Dawe [37], based on analysis of a large number of wide crosses, including oat, barley, and wheat (large centromere species) with sorghum, maize, pearl millet, adlay millet, and perennial ryegrass (small-centromere species), where genome elimination has been attributed to centromere size dimorphism, have proposed a centromere size model that suggests that centromere size has bearing on haploid production, as visible from failure in centromere function in the lines with small / weak centromere. Centromeres with defective or mutated CENH3 have a lower loading capacity and sport smaller centromeres, resulting in chromosome loss of the small-centromere parent in the intergenomic hybrid.

That the defective centromeres are lost in centromeremediated haploid production have been experimentally demonstrated by Ravi and Chan [27]. Using a modified version of CENH3 called "tailswap-CENH3" they were able to induce haploids at a very high frequency $(25-45 \%)$ in the Arabidopsis null mutant on account of non-functional centomeres (thus inactivating chromosome movement) of the tailswap parent that are eliminated. In fact the CENH3 mediated centromere manipulation has emerged as a powerful tool for uniparental chromosome elimination to realize haploid production for plant breeding applications $[5,28,38]$.

(d) Uniparental chromosome elimination and intergenomic balance: As mentioned above, the primary reason for selective elimination of a given genome during embryonic development in wide hybrids is on account of centromere dysfunction. But equally important is the spatial orientation of the participating genomes in the hybrid nucleus. It is observed that the genome destined to be eliminated is positioned in peripheral territory owing to weaker centromere function, slow condensation and shorter chromosomes to some extent $[19,21,25,31]$. Further, in the intergenomic hybrids where phenomenon of selective chromosomal elimination is widespread, e.g. in Hordeum, it is the ratio of the parental genomes in the hybrid combination that determines whether predominantly haploid or hybrid progeny are produced, and which genome is more likely to be eliminated, e.g. in a hybrid between tetraploid and diploid species of Hordeum, it is the diploid genome that would be eliminated as reported for several species of Hordeum [36]. Also, there is a hierarchy of species dominance in chromosome elimination [36]. Mukai et al. [26] further confirmed this assumption by demonstrating that the D-genome plays a critical role in the formation of haploid Aegilops tauschii through Imperata cylindrica mediated uniparental chromosome elimination.

Concluding Remarks: It could be conceptualized that in intergenomic hybrids, the partner genomes owe predisposed spatial territories; wherein peripheral genome incur subgenomic dominance, but prone to uniparental elimination owing centromere dysfunction. So what have we learned from the understanding of spatial orientation of genomes in the context of its implications in hybrid fixation and generation of new genomic states? Fixation of wide hybrids while maintaining the genomic territories of partner genomes also requires inter-genomic compatibility for coordinated cell division / kinetochore functionality, as well as minor readjustments in the genetic material through gene fractionation and gene expression. But in the incompatible hybrids one dominant genome could eliminate the other through 
inactivated centromeres of the latter. Such findings have enabled the development of haploid production technology through wide hybridization for its utilization in crop breeding programmes. Further, the observation that centromere dysfunction could result into chromosome elimination per se, has led to the development of technical advances for creating haploids at will through artificial manipulation of centromere function of one of the parents for its autoelimination during embryonic development, leaving only the haploids of choice at the end. This has opened a new era of development in plant breeding.

Acknowledgements Support received from INSA, New Delhi to UCL as Senior Scientist is gratefully acknowledged.

\section{Compliance with ethical standards}

Ethical statement Permission to reuse the figures has been obtained and original source is cited accordingly.

Conflict of interest Authors declare no conflict of interest.

\section{References}

1. Alger EI, Edger PP. One subgenome to rule them all: underlying mechanisms of subgenome dominance. Curr Opinion Plant Biol. 2020;54:108-13. https://doi.org/10.1016/j.pbi.2020.03.004.

2. Bennett MD, Finch RA, Barclay IR. The time rate and mechanism of chromosome elimination in Hordeum hybrids. Chromosoma. 1976;54:175-200.

3. Bennett ST, Bennett MD. Spatial Separation of Ancestral Genomes in the Wild Grass Milium montianum Parl.). Ann Bot. 1992;70:111-8.

4. Bird KA, VanBuren R, Puzey JR, Patrick P, Edger PP. The causes and consequences of subgenome dominance in hybrids and recent polyploids. New Phytologist. 2018;220:87-93. https://doi. org/10.1111/nph.15256.

5. Britt AB, Kuppu S. Cenh3: an emerging player in haploid induction technology. Front Plant Sci. 2016;7:357. https://doi.org/10.3389/ fpls.2016.00357.

6. Chan SWL. In a battle between parental chromosomes, a failure to reload. PNAS. 2011;108:13361-2. https://doi.org/10.1073/ pnas. 1110320108.

7. Corpet A, Almouzni G. Making copies of chromatin: the challenge of nucleosomal organization and epigenetic information. Trends Cell Biol. 2009;19:29-41.

8. Emery M, Willis MMS, Hao Y, Barry K, Oakgrove K, Peng Y, et al. Preferential retention of genes from one parental genome after polyploidy illustrates the nature and scope of the genomic conflicts induced by hybridization. PLoS Genet. 2018;14:e1007267. https:// doi.org/10.1371/journal.pgen.1007267.

9. Finch RA. Tissue-specific elimination of alternative whole parental genomes in one barley hybrid. Chromosoma. 1983;88:386-93.

10. Finch RA, Bennet MD. Mitotic and meiotic chromosome behaviour in new hybrids of Hordeum with Triticum and Secale. Heredity. 1980;44:201-9.

11. Finch RA, Bennett MD. The mechanism of somatic chromosome elimination in Hordeum. In: Kew Chromosome Conference II (ed. P.
B. Brandham, M. D. Bennett). 1983; pp. 147-154. London: George Allen \& Unwin

12. Gernand D, Rutten T, Varshney A, Rubtsova M, Prodanovic S, Bruss C, Kumlehn J, Matzk F, Houben A. Uniparental chromosome elimination at mitosis and interphase in wheat and pearl millet crosses involves micronucleus formation, progressive heterochromatinization, and DNA fragmentation. Plant Cell. 2005;17:2431-8. https:// doi.org/10.1105/tpc.105.034249.

13. Gleba YY, Parokonny A, Kotov V, Negrutiu I, Momot V. Spatial separation of parental genomes in hybrids of somatic plant cells. Proc Natl Acad Sci USA. 1987;84:3709-13.

14. Glombik M, Bačovský V, Hobza R, Kopecký D. Competition of Parental Genomes in Plant Hybrids. Front Plant Sci. 2020;11:200. https://doi.org/10.3389/fpls.2020.00200.

15. Han J, Zhou B, Shan W, Yu L, Wu W, Wang K. A and D genomes spatial separation at somatic metaphase in tetraploid cotton: evidence for genomic disposition in a polyploid plant. Plant $\mathbf{J}$. 2015;84:1167-77. https://doi.org/10.1111/tpj.13074.

16. Heslop-Harrison JS. Gene expression and parental dominance in hybrid plants. Development. 1990;108(Supplement):21-8.

17. Kim NS, Armstrong KC, Fedak G, Ho K, Park NI. A microsatellite sequence from the rice blast fungus (Magnaporthe grisea) distinguishes between the centromeres of Hordeum vulgare and $H$. bulbosum in hybrid plants. Genome. 2002;45:165-74.

18. Kolackova V, Pernickova K, Vrana J, Duchoslav M, Jenkins G, Phillips D, Turkosi E, Samajova O, Sedlarova M, Samaj J, et al. Nuclear disposition of alien chromosome introgressions into wheat and rye using 3D-FISH. Int J Mol Sci. 2019;20:4143. https://doi. org/10.3390/ijms20174143.

19. Komeda N, Chaudhary HK, Suzuki G, Mukai Y. Cytological evidence for chromosome elimination in wheat $\times$ Imperatacylindrica hybrids through fluorescence in situ hybridization. Genes Genet Syst. 2007;82:241-8.

20. Lavania UC, Srivastava S. Ploidy dependence of chromosomal variation in callus cultures of Hyoscyamus muticus L. Protoplasma. 1988;145:55-8.

21. Laurie DA, Bennett MD. The timing of chromosome elimination in hexaploid wheat $\times$ maize crosses. Genome. 1989;32:953-61.

22. Leitch AR, Schwarzacher T, Mosgöller WMD, Bennett MD, HeslopHarrison JS. Parental genomes are separated throughout the cell cycle in a plant hybrid. Chromosoma. 1991;101:206-13. https://doi. org/10.1007/BF00365152.

23. Lu F-H, McKenzie N, Gardiner L-J, Luo M-C, Hall A, Bevan MW. Reduced chromatin accessibility underlies gene expression differences in homologous chromosome arms of diploid Aegilopstauschii and hexaploid wheat. GigaScience. 2020;9(6):1-11. https://doi. org/10.1093/gigascience/giaa070.

24. Lysak MA, Berr A, Pecinka A, Schmidt R, McBreen K, Schubert I. Mechanisms of chromosome number reduction in Arabidopsis thaliana and related Brassicaceae species. Proc Natl Acad Sci USA. 2006;103:5224-9.

25. Mochida K, Tsujimoto H, Sasakuma T. Confocal analysis of chromosome behavior in wheat $\times$ maize zygotes. Genome. 2004;47:199205. https://doi.org/10.1139/g03-123.

26. Mukai Y, Okamoto G, Kiryu S, et al. The D-genome plays a critical role in the formation of haploid Aegilops tauschii through Imperata cylindrical mediated uniparental chromosome elimination. Nucleus. 2015;58:199-206. https://doi.org/10.1007/s13237-015-0151-2.

27. Ravi M, Chan SWL. Haploid plants produced by centromere-mediated genome elimination. Nature. 2010;464:615-8.

28. Ravi M, Marimuthu MPA, Tan EH, Maheshwari S, Henry IM, Marin-Rodriguez B, et al. A haploid genetics toolbox for Arabidopsis thaliana. Nat Commun. 2014;5:5334. https://doi.org/10.1038/ ncomms6334. 
29. Riera-Lizarazu O, Rines HW, Phillips RL. Cytological and molecular characterization of oat x maize partial hybrids. Theor Appl Genet. 1996;93:123-35.

30. Rodrigues JA. Zilberman D. Evolution and function of genomic imprinting in plants. Genes Dev. 2015;29:2517-31. https://doi. org/10.1101/gad.269902.115.

31. Sanei M, Pickering R, Kumke K, Nasuda S, Houben A. Loss of centromeric histone $\mathrm{H}_{3}\left(\mathrm{CENH}_{3}\right)$ from centromeres precedes uniparental chromosome elimination in interspecific barley hybrids. Proc Natl Acad Sci USA. 2011;108:E498-505. https://doi.org/10.1073/ pnas. 1103190108.

32. Schnable JC, Springer NM, Freeling M. Differentiation of the maize subgenomes by genome dominance and both ancient and ongoing gene loss. Proc Natl Acad Sci USA. 2011;108:4069-74.

33. Schwarzacher T, Heslop-Harrison JS, Anamthawat-Jonsson K, Finch RA, Bennett MD. Parental genome separation in reconstructions of somatic and premeiotic metaphases of Hordeum vulgare $\times H$. bulbosum. J Cell Sci. 1992;101:13-24.

34. Sharma AK. A new concept of speciation in plants. Caryologia. 1956;9:93-130.

35. Shaked H, Kashkush K, Ozkan H, Feldman M, Levy AA. Sequence elimination and cytosine methylation are rapid and reproducible responses of the genome to wide hybridization and allopolyploidy in wheat. Plant Cell. 2001;13:1749-59. https://doi.org/10.1105/ tpc. 010083 .

36. Subrahmanyam NC. Species dominance in chromosome elimination in barley hybrids. Curr Sci. 1982;51:28-31.

37. Wang N, Dawe RK. Centromere Size and Its Relationship to Haploid Formation in Plants. Mol Plant. 2018;11:398-406.

38. Wang N, Gent JI, Dawe RK. Haploid induction by a maize cenh3null mutant. SciAdv. 2021. https://doi.org/10.1126/sciadv.abe2299.

39. Wendel JF. The wondrous cycles of polyploidy in plants. Am J Bot. 2015;102:1753-6.

40. Yamamoto M. Molecular cytogenetic and epigenetic components of crossing barriers and peculiar reproduction ability in Allium hybrids. Nucleus. 2015;58:165-72. https://doi.org/10.1007/s1323 7-015-0153-0.

Publisher's Note Springer Nature remains neutral with regard to jurisdictional claims in published maps and institutional affiliations. 